\title{
SMOOTHNESS OF FUNCTIONS GENERATED BY RIESZ PRODUCTS
}

\section{PETER L. DUREN}

Riesz products are a useful apparatus for constructing singular functions with special properties. They have been an important source of counterexamples in various branches of analysis. We present here some relations between the structure of a Riesz product and the smoothness of the nondecreasing function it generates.

1. Preliminaries. Let $\phi(x)$ be a continuous complex-valued function defined on $0 \leqq x \leqq 2 \pi$. The modulus of continuity of $\phi$ is the function

$$
\omega(t)=\omega(t ; \phi)=\sup _{|x-y| \leqq t}|\phi(x)-\phi(y)| .
$$

$\phi$ belongs to the Lipschitz class $\Lambda_{\alpha}(0<\alpha \leqq 1)$ if $\omega(t)=O\left(t^{\alpha}\right)$ as $t \rightarrow 0$. Extend $\phi(x)$ to $-\infty<x<\infty$ by defining

$$
\phi(x)=k[\phi(2 \pi)-\phi(0)]+\phi(x-2 k \pi)
$$

for $2 k \pi \leqq x \leqq 2(k+1) \pi, k= \pm 1, \pm 2, \cdots$. If there is a constant $A$ such that

$$
|\phi(x+h)-2 \phi(x)+\phi(x-h)| \leqq A h, \quad h>0,
$$

we say that $\phi$ is of class $\Lambda^{*}$. For every $\alpha<1, \Lambda_{1} \subset \Lambda^{*} \subset \Lambda_{\alpha}$. In fact [5, p. 44], $\phi \in \Lambda^{*}$ implies $\omega(t)=O(t \log 1 / t)$.

Suppose now that $\phi(0)=\phi(2 \pi)$, and that

$$
\phi(x) \sim \sum_{n=-\infty}^{\infty} b_{n} e^{i n x}, \quad\left|b_{-n}\right|=\left|b_{n}\right| .
$$

LEMma 1. If

$$
\sum_{n=1}^{N} n\left|b_{n}\right|=O\left(N^{1-\alpha}\right), \quad 0<\alpha \leqq 1,
$$

then $\phi \in \Lambda_{\alpha}$. For $\alpha<1$, the converse is true if $b_{n} \geqq 0, n=1,2, \cdots$.

LEMMA 2. If

$$
\sum_{n=1}^{N} n\left|b_{n}\right|=O(\log N), \text { then } \omega(t ; \phi)=O(t \log 1 / t) .
$$

Presented to the Society, August 31, 1965; received by the editors November 26, 1964. 
LEMмA 3. If $\phi \in \Lambda^{*}$ and $b_{n} \geqq 0, n=1,2, \cdots$, then

$$
\sum_{n=1}^{N} n^{2}\left|b_{n}\right|=O(N) \text {. }
$$

These results can be verified (in greater generality) by well known elementary techniques, and the proofs are omitted. (Compare [1], where there is a proof of Lemma 3.)

2. Riesz products. I.et $n_{1}, n_{2}, \cdots$ be positive integers such that

$$
n_{j+1} / n_{j} \geqq q>3, \quad j=1,2, \cdots ;
$$

and let $a_{1}, a_{2}, \cdots$ be nonzero real numbers with $-1 \leqq a_{j} \leqq 1$. Then

$$
\mu_{k}=n_{1}+\cdots+n_{k} \leqq n_{k}\left(1+1 / q+\cdots+1 / q^{k-1}\right)<\frac{q}{q-1} n_{k},
$$

so that

$$
(1-1 / q) \mu_{k}<n_{k} \leqq \mu_{k} .
$$

Now consider the trigonometric polynomials

$$
p_{k}(t)=\prod_{j=1}^{k}\left(1+a_{j} \cos n_{j} t\right)=1+\sum_{\nu=1}^{\mu_{k}} c_{\nu} \cos \nu t .
$$

The $c_{\nu}$ are constructed from the $a_{j}$ through the identity $\cos n t \cos m t$ $=\frac{1}{2}[\cos (n+m) t+\cos (n-m) t]$. Specifically, $c_{\nu}=0$ unless $\nu$ is of the form

$$
\nu=n_{j_{1}} \pm n_{j_{-1}} \pm \cdots \pm n_{j_{1}}
$$

where $1 \leqq j_{1}<j_{2}<\cdots<j_{\text {. }}$. If $\nu$ has the form (2), then

$$
c_{\nu}=2^{-s+1} a_{j_{1}} a_{j_{2}} \ldots a_{j_{e}} .
$$

Thus there are $2^{s-1}$ coefficients with the same value (3), corresponding to the $2^{-1}$ distinct integers $\nu$ in a particular set (2). We shall make use of the relations

$$
\begin{aligned}
1+\sum_{v=1}^{\mu_{k}}\left|c_{\nu}\right| & =\prod_{j=1}^{k}\left(1+\left|a_{j}\right|\right) ; \\
\Gamma_{k}-\Gamma_{k-1} & =n_{k} a_{k} \prod_{j=1}^{k-1}\left(1+a_{j}\right), \quad \text { where } \Gamma_{k}=\sum_{v=1}^{\mu_{k}} \nu c_{\nu .}
\end{aligned}
$$

To prove (4), we need only note that

$$
p_{k}(0)=1+\sum_{\nu=1}^{\mu_{k}} c_{\nu}=\prod_{j=1}^{k}\left(1+a_{j}\right)
$$


and that the sequence $\left\{\left|a_{j}\right|\right\}$ generates the sequence $\left\{\left|c_{\nu}\right|\right\}$. The sum $\Gamma_{k}-\Gamma_{k-1}$ is actually extended only over the integers of the form (2), where $j_{s}=k$. On the other hand, the sum of all $\nu$ in any particular set (2) is obviously $2^{-1} n_{k}$. Thus

$$
\sum \nu c_{\nu}=n_{k} a_{k} a_{j_{1}} \cdots a_{j_{g-1}}
$$

if the sum is extended over a particular set (2). Summing over all sets (2), we obtain (5).

It can be shown $[3],[5$, p. 208] that

$$
F(x)=\lim _{k \rightarrow \infty} \int_{0}^{x} p_{k}(t) d t
$$

exists for all $x, 0 \leqq x \leqq 2 \pi$, and is a continuous nondecreasing function.

THEOREM 1. If

$$
\prod_{j=1}^{k}\left(1+\left|a_{j}\right|\right)=O\left(n_{k}^{1-\alpha}\right), \quad 0<\alpha \leqq 1,
$$

then $F \in \Lambda_{\alpha}$. For $\alpha<1$, the converse is true if all $a_{j}>0$.

THEOREM 2. If

$$
\prod_{j=1}^{k}\left(1+\left|a_{j}\right|\right)=O\left(\log n_{k}\right)
$$

then $\omega(t ; F)=O(t \log 1 / t)$.

Proofs. Consider the function

$$
\Phi(x)=i[F(x)-x] \sim \sum_{\nu=-\infty}^{\infty} b_{\nu} e^{i \nu x}, \quad b_{\nu}=c_{|\nu|} / 2 \nu .
$$

In view of (1) and (4), the relation (6) implies

$$
1+\sum_{\nu=1}^{\mu_{k}}\left|c_{\nu}\right| \leqq A \mu_{k}^{1-\alpha}, \quad k=1,2, \cdots
$$

where $A$ is a constant. Given an arbitrary positive integer $N$, choose $k$ such that $\mu_{k-1}<N \leqq \mu_{k}$. Then

$$
\begin{gathered}
|\phi(x+h)-2 \phi(x)+\phi(x-h)| \leqq A h, \quad h>0, \\
1+\sum_{\nu=1}^{N}\left|c_{\nu}\right| \leqq \prod_{j=1}^{k}\left(1+\left|a_{j}\right|\right)=\left(1+\left|a_{k}\right|\right)\left[1+\sum_{\nu=1}^{\mu_{k-1}}\left|c_{\nu}\right|\right] \\
\leqq 2 A \mu_{k-1}^{1-\alpha} \leqq 2 A N^{1-\alpha}
\end{gathered}
$$

This for all $N$ implies $\Phi \in \Lambda_{\alpha}$, by Lemma 1 ; hence $F \in \Lambda_{\alpha}$. Theorem 2 
follows similarly from Lemma 2 . The converse in Theorem 1 is immediate from Lemma 1 , since $a_{j}>0$ implies $c_{p} \geqq 0$.

We mention one application of Theorem 1 . If $\left|a_{j}\right| \leqq M \leqq 1$, then

$$
F \in \Lambda_{\alpha}, \quad \alpha=1-\log (1+M) / \log q,
$$

and this statement is best possible. For $M=1$, (8) was noted by Zygmund [5, p. 230, problem 23].

The function $F$ generated by a Riesz product is either purely singular $\left(F^{\prime}(x)=0\right.$ a.e.) or absolutely continuous. In fact, there is the following basic theorem, due to Zygmund [4, p. 440], [5, p. 209].

Theorem A. The function $F$ is singular if and only if $\sum a_{j}^{2}=\infty$. If $\sum a_{j}^{2}<\infty$, then $F$ is absolutely continuous and $F^{\prime} \in L^{2}$.

The second statement follows from the fact that convergence of $\sum a_{j}^{2}$ implies convergence of $\sum c_{\nu}^{2}$. Zygmund pointed this out [4, p. 438], but with no explicit proof. We give here a proof suggested by A. L. Shields. In fact, it is just as easy to prove something more general: For any $p>0, \sum\left|c_{\nu}\right|^{p}<\infty$ if and only if $\sum\left|a_{j}\right|^{p}<\infty$. Indeed,

$$
\begin{aligned}
\sum_{\nu=1}^{\infty}\left|c_{\nu}\right|^{p}= & \sum_{i}\left|a_{i}\right|^{p}+2 \sum_{i<j}\left(2^{-1}\left|a_{i} a_{j}\right|\right)^{p}+2^{2} \sum_{i<j<k}\left(2^{-2}\left|a_{i} a_{j} a_{k}\right|\right)^{p} \\
& +\cdots \\
= & 2^{p-1} \coprod_{j=1}^{\infty}\left(1+2^{1-p}\left|a_{j}\right|^{p}\right)-2^{p-1} .
\end{aligned}
$$

But the infinite product converges if and only if $\sum\left|a_{j}\right|^{p}<\infty$.

3. The class $\Lambda^{*}$. In a recent investigation of certain pathological domains in the complex plane [1], it was of key importance to construct a singular nondecreasing function of class $\Lambda^{*}$. As a consequence of the theory, it was pointed out that a certain geometric example of Keldyš and Lavrentiev implies the existence of such functions. G. Piranian and J.-P. Kahane [2] later gave constructions based directly on the definition of $\Lambda^{*}$. It is natural to ask whether such a function can be constructed by means of a Riesz product. The example $a_{j}=1, n_{j}=2^{2^{j}}$ shows (by Theorem A and Theorem 2) that Riesz products are capable of producing singular functions with $\omega(t)$ $=O(t \log 1 / t)$. This would seem to be encouraging, because $\Lambda^{*}$ functions must have this degree of smoothness. The answer, however, is negative.

THEOREM 3. No Riesz product can generate a function $F$ which is both singular and of class $\Lambda^{*}$. 
PRoof. If $F \in \Lambda^{*}$, the same is true of the function $\Phi$ given in (7). Suppose first that $a_{j}>0, j=1,2, \cdots$. Then all $c_{p} \geqq 0$, and by Lemma 3,

$$
\sum_{v=1}^{N} \nu c_{\nu}=O(N)
$$

Taking (5) and (1) into account, we conclude that

$$
a_{k} \prod_{j=1}^{k-1}\left(1+a_{j}\right) \leqq C, \quad k=2,3, \cdots
$$

The next step is to show that (9) implies $\sum a_{j}^{2}<\infty$, which (by Theorem A) will finish the proof in the case $a_{j}>0$. For this we present an ingenious argument due to $\mathrm{D}$. J. Newman (private communication). It rests on the two simple inequalities

$$
\begin{aligned}
e^{x / 2} & \leqq 1+x, \\
x & \leqq 3\left(1-e^{-x / 2}\right), \quad 0 \leqq x \leqq 1 .
\end{aligned}
$$

From (9) and (10) we find

$$
a_{k} \leqq C \exp \left\{-\frac{1}{2} \sum_{j=1}^{k-1} a_{j}\right\} .
$$

Without harm, we may suppose $C \geqq 3$. Then, by (11),

$$
a_{k} \leqq C\left[1-\exp \left\{-\frac{1}{2} a_{k}\right\}\right] \text {. }
$$

Multiplication of (12) and (13) gives

$$
a_{k}^{2} \leqq C^{2}\left[\exp \left\{-\frac{1}{2} \sum_{j=1}^{k-1} a_{j}\right\}-\exp \left\{-\frac{1}{2} \sum_{j=1}^{k} a_{j}\right\}\right] .
$$

If we sum (14) over $2 \leqq k \leqq m$, the dominant series telescopes, and we find

$$
\sum_{k=2}^{m} a_{k}^{2} \leqq C^{2}, \quad m=2,3, \cdots .
$$

Finally, suppose that $F(x) \in \Lambda^{*}$ is generated by a Riesz product whose coefficients $a_{j}$ have arbitrary signs. Let $G(x)$ be generated by the Riesz product with coefficients $\epsilon_{j}=\operatorname{sgn} a_{j}$ and the same frequencies $n_{j}$. Set $f(x)=F(x)-x$ and $g(x)=G(x)-x$; then the convolution 


$$
h(x)=\frac{1}{2 \pi} \int_{0}^{2 \pi} f(x-t) d g(t) \in \Lambda^{*} .
$$

Furthermore, if

$$
d f(x) \sim \sum_{\nu=1}^{\infty} c_{\nu} \cos \nu x \text { and } d g(x) \sim \sum_{\nu=1}^{\infty} \gamma_{\nu} \cos \nu x
$$

then

$$
d h(x) \sim \sum_{\nu=1}^{\infty} d_{\nu} \cos \nu x, \text { where } d_{\nu}=\frac{1}{2} c_{\nu} \gamma_{\nu} .
$$

But for $\nu$ of the form (2), $c$, is given by (3), and

$$
\gamma_{\nu}=2^{-s+1} \epsilon_{j_{1}} \epsilon_{j_{2}} \cdots \epsilon_{j_{\bullet}}
$$

Thus

$$
d_{\nu}=2^{-\imath+1} 2^{-\bullet}\left|a_{j_{1}} a_{j_{2}} \cdots a_{j_{\ell}}\right| .
$$

This means that the function $H(x)=h(x)+x \in \Lambda^{*}$ is generated by a Riesz product with positive coefficients $\left|a_{j}\right| / 2$. Therefore, by what we have already proved, $\sum a_{j}^{2}<\infty$.

AckNowledgments. This paper owes much to discussion with H. S. Shapiro and A. L. Shields. I am indebted to N. T. Varopoulos for suggesting the use of convolutions in the proof of Theorem 3. Finally, I wish to express my gratitude to the Alfred P. Sloan Foundation for its generous support.

\section{REFERENCES}

1. P. L. Duren, H. S. Shapiro and A. L. Shields, Singular measures and domains not of Smirnov type, Duke Math. J. (to appear).

2. G. Piranian, Two monotonic, singular uniformly almost smooth functions, Duke Math. J. (to appear).

3. F. Riesz, Über die Fourierkoeffizienten einer stetigen Funktion von beschränkter Schwankung, Math. Z. 2 (1918), 312-315.

4. A. Zygmund, On lacunary trigonometric series, Trans. Amer. Math. Soc. 34 (1932), 435-446.

5. - Trigonometric series, Vol. I, 2nd ed., Cambridge Univ. Press, Cambridge, 1959.

UNIVERSITY OF MiCHIGAN 